\title{
Insulation Coordination for a Modular Multilevel Converter Prototype
}

A. Christe, E. Coulinge, and D. Dujic

This material is posted here with permission of the IEEE. Such permission of the IEEE does not in any way imply IEEE endorsement of any of EPFL's products or services. Internal or personal use of this material is permitted. However, permission to reprint / republish this material for advertising or promotional purposes or for creating new collective works for resale or redistribution must be obtained from the IEEE by writing to pubs-permissions@ieee. org. By choosing to view this document, you agree to all provisions of the copyright laws protecting it. 


\title{
Insulation Coordination for a Modular Multilevel Converter Prototype
}

\author{
Alexandre Christe, Emilien Coulinge, Drazen Dujic \\ Power Electronics Laboratory \\ École Polytechnique Fédérale de Lausanne - EPFL \\ Station 11, CH-1015 Lausanne \\ Email: alexandre.christe@epfl.ch,emilien.coulinge@epfl.ch,drazen.dujic@epfl.ch \\ URL: http://pel.epfl.ch
}

\section{Acknowledgments}

This work is part of the Swiss Competence Centers for Energy Research (SCCER) initiative which is supported by the Swiss Commission for Technology and Innovation (CTI) with focus on Future Swiss Electrical Infrastructure (FURIES).

\section{Keywords}

$<<$ Modular Multilevel Converters (MMC) $>>,<<$ Design $>>,<<$ Insulation $>>,<<$ FEM calculation $>>$.

\begin{abstract}
This paper provides a detailed overview of the dielectric design and insulation coordination applied to a medium voltage modular multilevel converter prototype. The complete system has ratings of $0.5 \mathrm{MVA}$ and is designed for connection to a $10 \mathrm{kV}_{\mathrm{dc}}$ supply with a system voltage of $6.6 \mathrm{kV}_{\mathrm{ac}}$. The choice of air as insulating and cooling medium requires careful considerations regarding the clearance and creepage distances with respect to the selected materials. The design considerations from the submodule to the cabinet level are presented in the paper, considering safety standards and their requirements for a selected over-voltage category and pollution degree. The final dielectric design is verified experimentally with dielectric ac withstand test and partial discharge measurements.
\end{abstract}

\section{Introduction}

Following its introduction, the modular multilevel converter (MMC) [1] has been gaining in popularity and is nowadays considered for various applications, predominantly those with medium or high voltages. Even though MMC is commercialized by the industry, there are still many open research topics and optimization opportunities. While numerous MMC prototypes have been realized and reported in academia, the majority of them are laboratory scale prototypes rated for low voltages (LVs) and powers, thus effectively avoiding a need for development of technologies that would normally have to be considered for medium voltage (MV) converters (e.g. insulation coordination, auxiliary power supply, protection, etc.). There are some notable exceptions, such as the work reported in [2], while data on various other industrial designs are not easily obtainable.

This paper describes the designs applied during the development of a medium voltage MMC prototype, primarily focusing on the dielectric aspects and insulation coordination. The MMC prototype has ratings of $0.5 \mathrm{MVA}$ and is designed for connection to $10 \mathrm{kV}$ on the MVDC side and $400 \mathrm{~V}$ on the LVAC side. The discrete branch inductors and the line frequency transformer are combined in a dual-primary transformer, resulting in the so-called galvanically isolated modular converter (GIMC) [3, 4]. Nevertheless, the transformer primary windings are rated for $6.6 \mathrm{kV}$, similarly to the classical MMC. During the design phase, $1.2 \mathrm{kV}$ rated IGBTs are selected, resulting in 16 submodules (SMs) per branch, or 96 for the whole converter. 
Considering the connection to $10 \mathrm{kV}_{\mathrm{dc}}$, several design constraints have to be satisfied, related to the choice of cooling medium, the insulation concept, clearance and creepage distances, as well as selection of suitable materials and arrangements of the different sub-assemblies that contribute actively to meeting the standards. Various norms and standards have been consulted (e.g. UL 840 [5], IEC 61800-5-1 [6]) to identify the applicable requirements and constraints, keeping in mind the laboratory use of the equipment, the presence of already installed protection equipments and controlled environmental conditions. Design tools, such as finite element modeling (FEM) ${ }^{1}$, are used for simulation and design optimization, prior to the experimental verification with high voltage test equipment.

\section{System Level Considerations}

The schematic of the complete laboratory system is shown in Fig. 1 . The $10 \mathrm{kV}_{\mathrm{dc}}$ supply is provided through a laboratory installed step-up transformer (vector group Yd11y0) and a 12-pulse rectifier, while the $400 \mathrm{~V}_{\mathrm{ac}}$ output connection is available for load or grid connections after the dual-primary transformer. Alternatively, a direct access to the MVAC output is possible by substitution of the dual-primary transformer with conventional branch inductors. The complete prototype is realized in a modular fashion and partitioned into five cabinets. The work presented in this paper is mainly focused on the three cabinets where SMs are installed, and discussion related to the other parts of the system are omitted. Considering the IEC 61800-5-1 standard, the nature of the installation with no direct connection or exposure of the circuit to the direct transient over-voltages and the choice of forced air cooling with air from indoor, over-voltage category (OVC) 1 and pollution degree (PoD) 1 should suffice design. Nevertheless, as this is a laboratory prototype for multipurpose use, more stringent OVC2 and PoD2 are considered throughout the design. Four insulation zones are identified, characterized by different working voltages, materials and requirements for insulation coordination. They are graphically presented in Fig. 2.

Zone 1: insulation coordination inside the enclosure of the SM. The nominal SM capacitor voltage $\left(V_{C, \text { nom }}\right)$ is $650 \mathrm{~V}_{\mathrm{dc}}$, with maximum voltage of $900 \mathrm{~V}_{\mathrm{dc}}\left(V_{C, \text { max }}\right)$ before trigger of the protective $\mathrm{SM}$ bypass. The system voltage is $1 \mathrm{kV}_{\mathrm{ac}}$.

Zone 2: insulation coordination between the SMs within a branch. Due to the matrix layout of the SMs, the maximum voltage between two adjacent SMs is different on the horizontal and vertical axes. It is equal to $V_{C \text {, max }}$ and $4 V_{C \text {, max }}$, respectively. They correspond to system voltages of $1 \mathrm{kV}_{\mathrm{ac}}$ (no interpolation allowed) and $3.6 \mathrm{kV}_{\mathrm{ac}}$.

Zone 3: insulation coordination between a branch and the surrounding cabinet (at ground (GND) potential). This also includes any passages made available for cables routing. The system voltage considered for insulation coordination is $6.6 \mathrm{kV}_{\mathrm{ac}}$.

Zone 4: insulation coordination related to the low voltage circuits, such as control boards or auxiliary power supply systems. The nominal voltage is $400 \mathrm{~V}_{\mathrm{ac}}$.

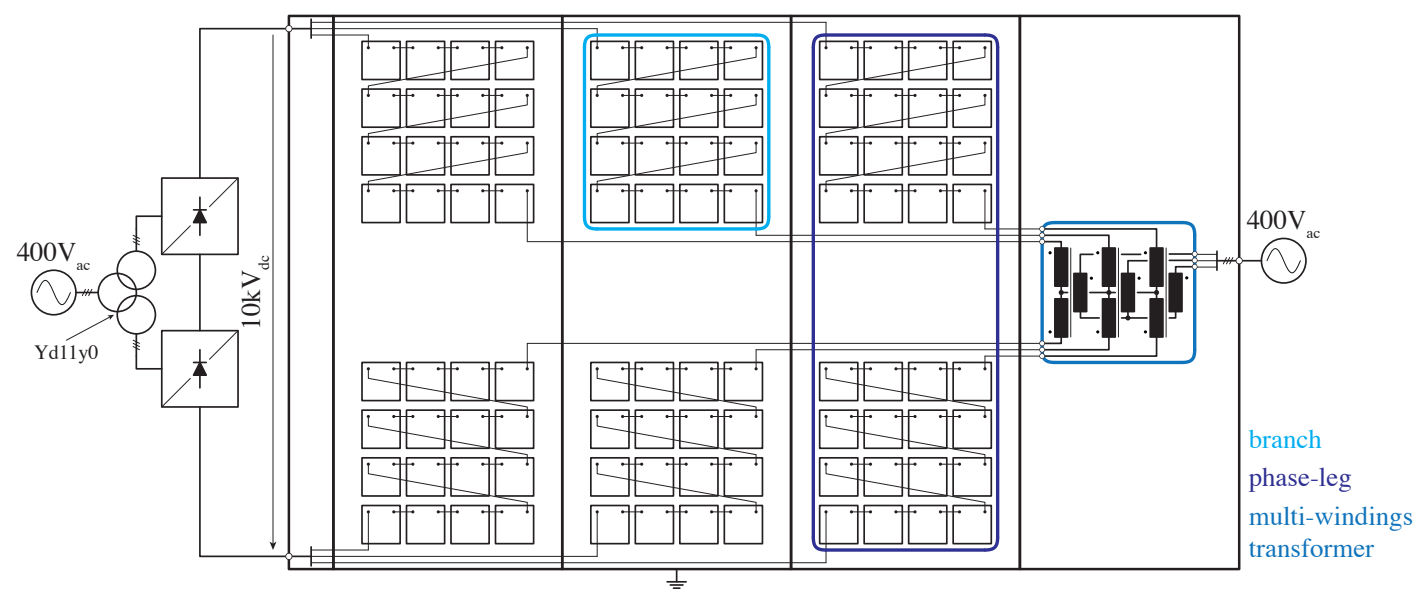

Fig. 1: MMC prototype converter simplified layout and connection to the laboratory infrastructure.

${ }^{1}$ All FEM simulations are performed with the software ANSYS Maxwell from the company ANSYS Inc. 


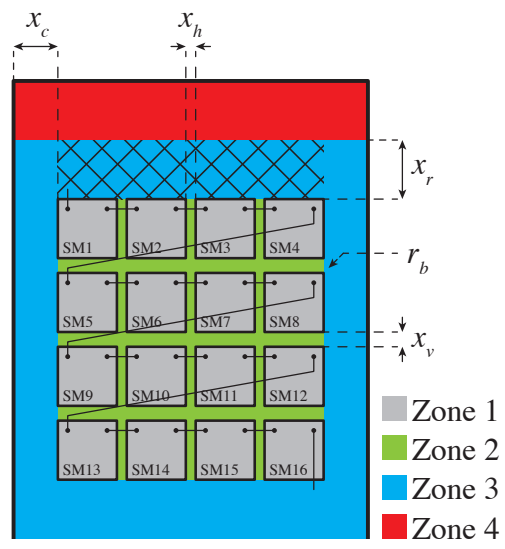

(a)

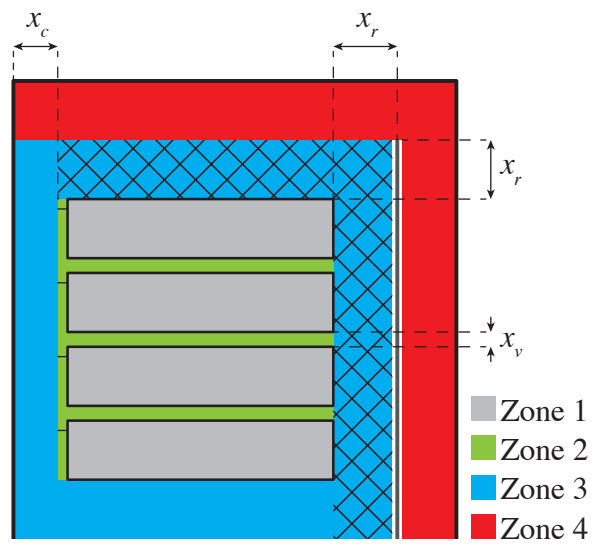

(b)

Fig. 2: (a) front view and (b) side view for the definition of the different insulation coordination zones inside the cabinet. The relevant distances are also indicated, and $x$ should be substituted with $d_{\mathrm{L}}$ and $d_{\mathrm{C}}$ for clearance and creepage distances, respectively. The hatched areas correspond to required reinforced isolation.

\section{Zone 1 - MMC submodule}

The basic building block is the MMC SM and its early prototype is shown in Fig. 3. It features a Power $P C B$ as the interface between the power semiconductors and the SM capacitor bank. Copper bus-bars are used as power connections towards the other SMs. The IGBT module (1200 V, 50 A) is in full-bridge configuration (the SM can be mechanically reconfigured either in half-bridge or in full-bridge configuration) and is mounted on a heat-sink together with the fast thyristor bypass module (the slow bypass bi-stable relay is mounted on top). The capacitor bank is rated for $800 \mathrm{~V}$ with $2.1 \mathrm{mF}$ and is realized with six electrolytic capacitors. On top, an Adapter PCB hosts the SM DSP, gate drivers, measurements processing circuits, protection logic and optical fiber links for communication. The auxiliary power supply is provided through an inductive power transfer (IPT) system, which is not explicitly shown.

Each SM is enclosed in a metallic box, providing shielding, protection and shaping of the surrounding E-field. Each SM defines the enclosure potential, which is referenced to the negative capacitor bank terminal. It effectively floats on a potential with respect to the external system GND. As the SM is a low voltage circuit, both UL 840 and IEC 61800-5-1 are considered. Yet, with IEC 61800-5-1 being safety standards, it is more relevant for the design outside the SM. By considering a maximum expected voltage inside the SM of $V_{C, \max }$, OVC2 and FR-4 for printed circuit board (insulating material group IIIa, comparative tracking index (CTI) between 175 and 200), the minimum clearance and creepage distances are determined and applied, respectively (cf. Table I). The complete SM assembly is placed inside a custom protruded aluminum enclosure, designed to provide a mechanical support, a defined air channel, and a shaping of the E-field at the corners below the critical levels considering air insulation inside the

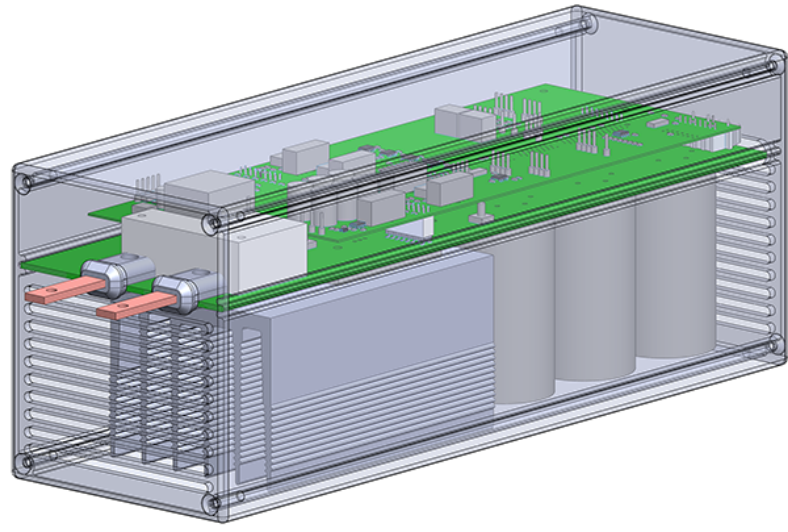

(a)

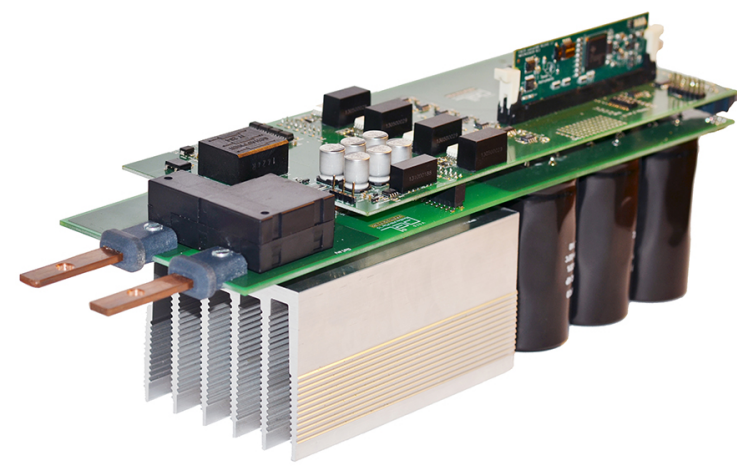

(b)

Fig. 3: (a) 3D CAD rendering within enclosure and (b) prototype of a SM. 


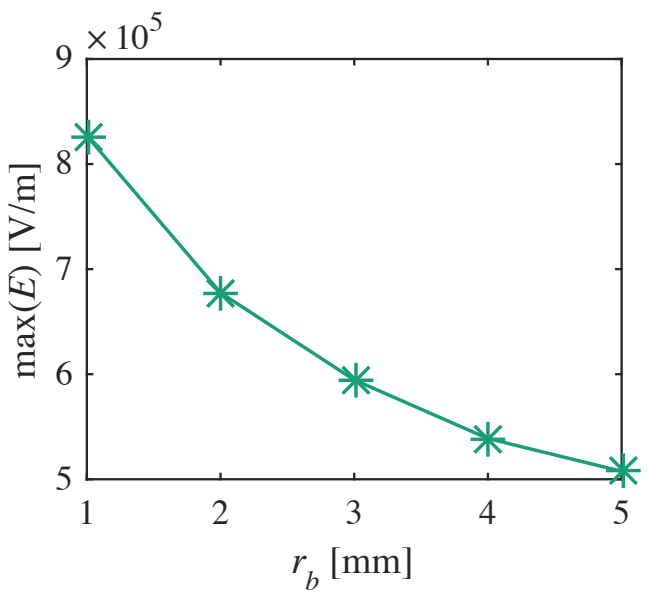

(a)
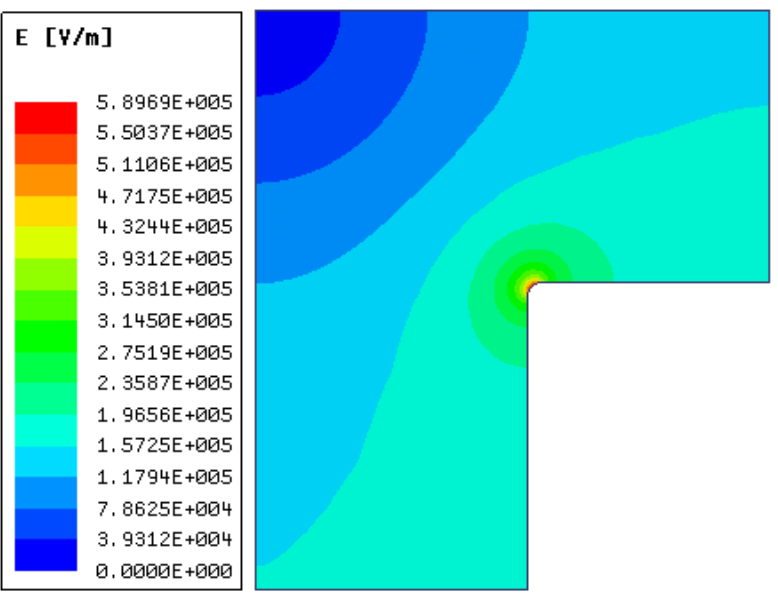

(b)

Fig. 4: (a) evolution of $\max (E)$ when $r_{b}$ is varied from $1 \mathrm{~mm}$ to $5 \mathrm{~mm}$ for the top corner and (b) FEM E-field magnitude with $r_{b}=3 \mathrm{~mm}$ plus $62 \mathrm{~mm}$ to ground.

cabinet (e.g. considering maximum theoretical value of $3 \mathrm{kV} / \mathrm{mm}$ ). In Fig. $4 \mathrm{a}$, the enclosure corner radius $r_{b}$ is varied from $1 \mathrm{~mm}$ to $5 \mathrm{~mm}$ and multiple FEM simulations are performed to verify that the maximum E-field is below the critical value of $3 \mathrm{kV} / \mathrm{mm}$. The E-field magnitude for the final design with $r_{b}=3 \mathrm{~mm}$ is presented in Fig. $4 \mathrm{~b}$.

Table I: Summary of zone 1 isolation distances requirements.

\begin{tabular}{|l|c|}
\hline Variable & Minimal value $[\mathrm{mm}]$ \\
\hline$d_{\mathrm{L}, S M}$ & 3 \\
$d_{\mathrm{C}, S M}$ & 6.3 \\
\hline
\end{tabular}

\section{Zone 2 - MMC branch}

All 16 SMs of one branch are arranged into a $4 \times 4$ matrix, as shown in Fig. 2a. Due to this arrangement, the horizontal and vertical potential differences during operation between two adjacent SMs are not the same. As previously stated, they equal $V_{C, \max }$ and $4 V_{C, \max }$, respectively. This has been taken into account during the design of the SM enclosure and for the choice of the clearance distances. Even though IEC61800-5-1 considers non-homogeneous E-field distribution, care is taken to avoid sharp edges on the enclosure and avoid excessive E-field values (namely mitigate the peak effect). Additionally, to provide mechanical support to the SMs, easy installation and quick removal from the cabinet, the SMs are grouped in a drawer alike sub-assembly that consists of four SMs, as shown in Fig. 5. The material used for the drawer is a melamine / paper-based laminate ${ }^{2}$ (insulating material group I, CTI of 600), which was helpful to reduce creepage distances in the design. As each drawer has metallic sliding rails for easy mounting, the insulation coordination must consider not only the distances relevant for the Zone 2, but also nearby Zone 3.

The constraints are presented in Table II and have been verified using FEM simulations. To define the exact shape of the SM enclosure, the corner's radius has been optimized to shape the surrounding E-field. At the same time, horizontal and vertical distances for mechanical integration have been verified with respect to the maximum E-field values observed at relevant points. FEM simulations have been carried out with an excitation voltage equivalent to $1.1 \mathrm{kV}_{\mathrm{ac}}$ and $4 \mathrm{kV}_{\mathrm{ac}}$ for horizontal and vertical clearances, respectively, according to the IEC61800-5-1 standard for AC test requirements. FEM simulation results are shown in Fig. 6a, from where it can be seen that the maximum values of E-field are substantially below the air breakdown level when $r_{b}$ is varied from $1 \mathrm{~mm}$ to $5 \mathrm{~mm}$. The E-field magnitude for the final design with $r_{b}=3 \mathrm{~mm}$ is presented in Fig. $6 \mathrm{~b}$.

${ }^{2}$ Melaminkaschiertes Hartpapier (MKHP). 


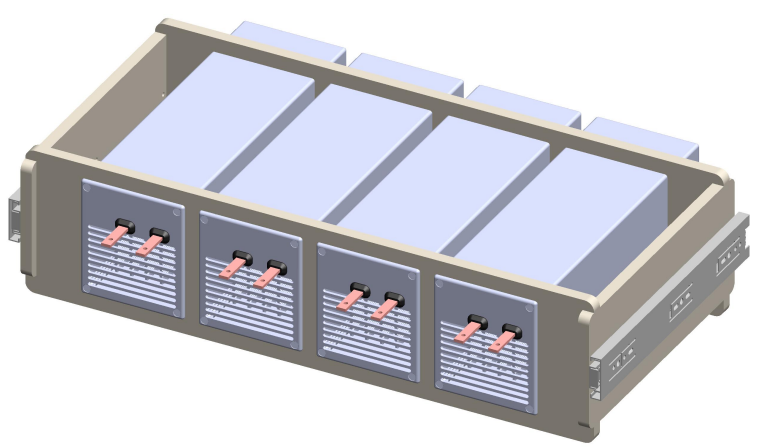

(a)

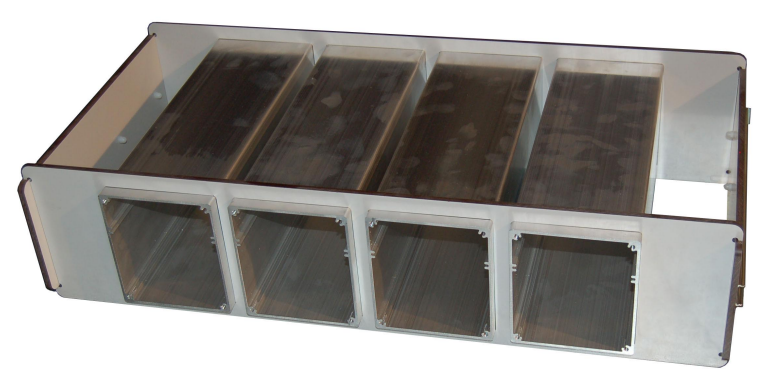

(b)

Fig. 5: (a) CAD of the drawer sub-assembly containing four SMs and (b) realization.

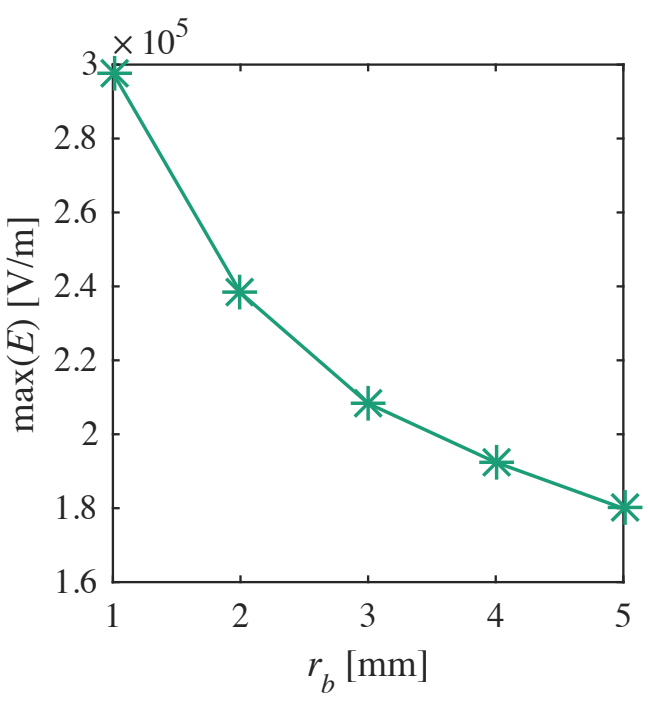

(a)

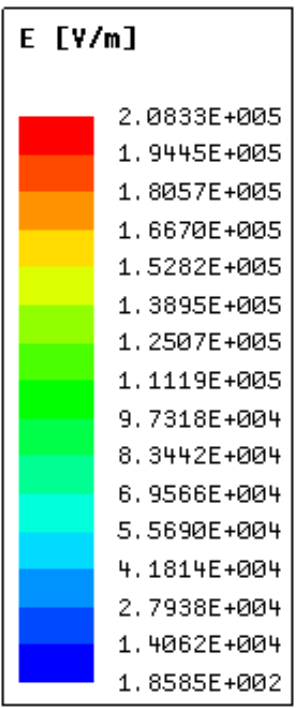

(b)

Fig. 6: (a) evolution of $\max (E)$ when $r_{b}$ is varied from $1 \mathrm{~mm}$ to $5 \mathrm{~mm}$ and (b) FEM simulation of the E-field magnitude around four SM enclosures with $r_{b}=3 \mathrm{~mm}$.

Table II: Summary of zone 2 isolation distances requirements.

\begin{tabular}{|l|c|}
\hline Variable & Minimal value $[\mathrm{mm}]$ \\
\hline$d_{\mathrm{L}, h}$ & 6.8 \\
$d_{\mathrm{C}, h}$ & 3.2 \\
$d_{\mathrm{L}, v}$ & 30 \\
$d_{\mathrm{C}, v}$ & 12.5 \\
\hline
\end{tabular}

\section{Zone 3 - MMC phase-leg}

Since various metallic parts of the cabinet are at the GND potential with respect to the floating SMs, the full system voltage rating must be taken into account. A basic insulation is sufficient for most of the Zone 3 , considering the space between the branch assembly (live parts) and cabinet side walls at GND. The metallic sliding rails, mounted on the sides of each drawer, are the closest GND points from the live parts (SMs). However the presence of low voltage circuits, such as an IPT supply on the back side between the SMs and the cabinet wall or a controller on top of the cabinet, requires reinforced or double insulation to be deployed. This is illustrated in Fig. 2, where the hatched areas of Zone 3 are those where reinforced insulation is required.

The constraints are presented in Table III and have been verified using FEM simulations. Theoretically, there are $2^{16}=65536$ cases that should be tested, however this number is way too large. The simulation 


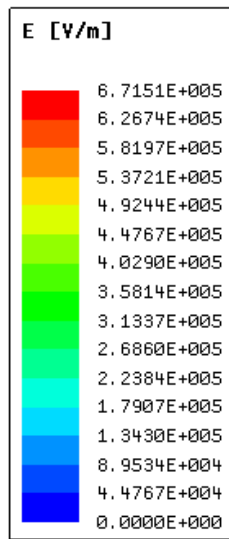

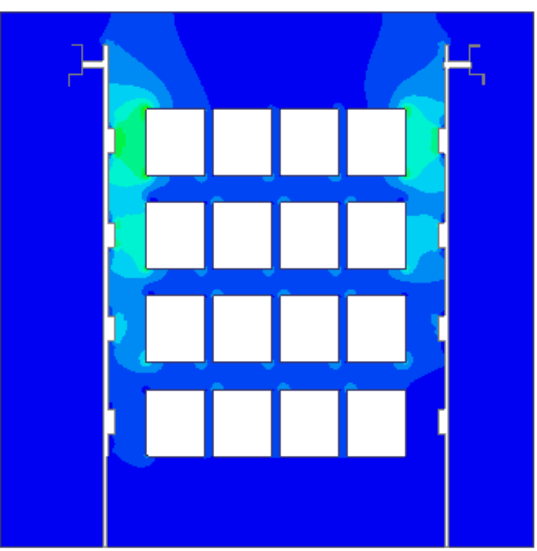

(a)

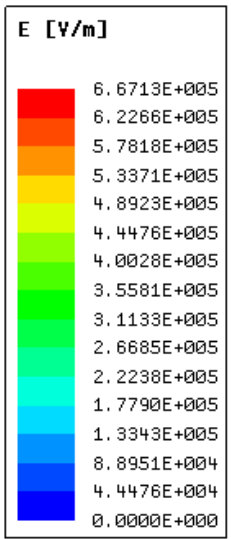

$.0000 \mathrm{E}+000$

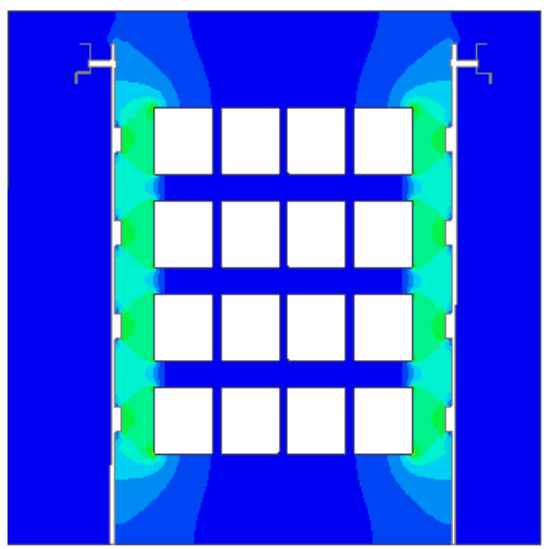

(b)

Fig. 7: (a) FEM simulation for E-field magnitude with 16 SMs inserted (1 out of $2^{16}$ combinations) and (b) FEM simulation for E-field magnitude with 16 SMs bypassed (all clamped to the positive DC terminal). Only the upper branch is displayed, as similar results would be observed in the lower one.

results are shown in Fig. 7 under the condition of the complete branch assembly (16 SMs) only for symmetry reason, with an excitation voltage equivalent to $11 \mathrm{kV}_{\mathrm{ac}}$ (level required for $\mathrm{AC}$ test in case of basic insulation). As it can be seen, the values of the E-field observed near the mounting rails are sufficiently below the air breakdown level.

Table III: Summary of zone 3 isolation distances requirements.

\begin{tabular}{|l|c|}
\hline Variable & Minimal value $[\mathrm{mm}]$ \\
\hline$d_{\mathrm{L}, c}$ & 60 \\
$d_{\mathrm{C}, c}$ & 50 \\
$d_{\mathrm{L}, r}$ & 102 \\
\hline
\end{tabular}

\section{Final design}

The manufacturing capabilities, required distances for handling as well as internal cabinet dimensions influenced the final design, which is summarized in Table IV. The selected cabinet $(1970 \mathrm{~mm} \times 800 \mathrm{~mm}$ $\times 800 \mathrm{~mm}$ ) is equipped with a meshed front door and an air chimney at the back for forced air cooling. Details about the complete cooling concept are presented in [7].

Table IV: Mechanical design summary. The distances concerning the PCB part of the SM are of less relevance and were intentionally omitted in this table.

\begin{tabular}{|l|c|c|}
\hline Variable & Minimal value $[\mathrm{mm}]$ & Actual design value $[\mathrm{mm}]$ \\
\hline$r_{b}$ & & 3 \\
$d_{\mathrm{L}, h}$ & 6.8 & 15 \\
$d_{\mathrm{C}, h}$ & 3.2 & 15 \\
$d_{\mathrm{L}, v}$ & 30 & 50 \\
$d_{\mathrm{C}, v}$ & 12.5 & 275 \\
$d_{\mathrm{L}, c}$ & 60 & 81.5 \\
$d_{\mathrm{C}, c}$ & 50 & 93 \\
$d_{\mathrm{L}, r}$ & 102 & 120 \\
\hline
\end{tabular}




\section{Experimental verification}

To verify the design, several tests are performed using the available high voltage test setup for ac dielectric withstand and partial discharge (PD) measurements rated for $100 \mathrm{kV}, 20 \mathrm{kVA}$ [8]. The measurements were performed with Omicron MPD600 [9]. The inside of the Faraday cage is shown in Fig. 8, with the high voltage step-up transformer, damping resistor, parallel capacitor to the device under test, which is the phase-leg MMC cabinet. The device was calibrated at $1 \mathrm{pC}$ and the setup has a noise level below $100 \mathrm{fC}$. For each test, the voltage profile follows Fig. 9 with the appropriate $U_{\mathrm{PD}}$. Please note that $U_{\mathrm{PD}}$ is equal to the recurring voltage of the system.

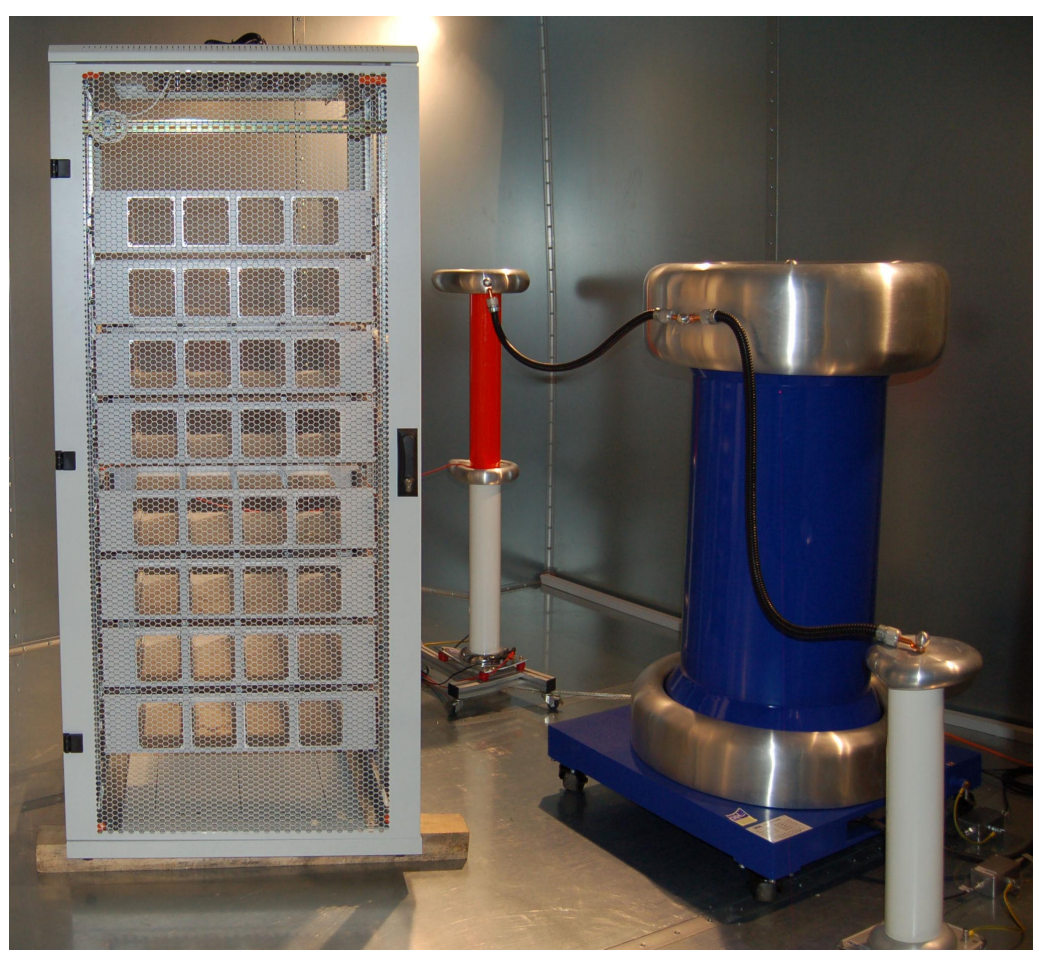

Fig. 8: PD test setup inside the Faraday cage.

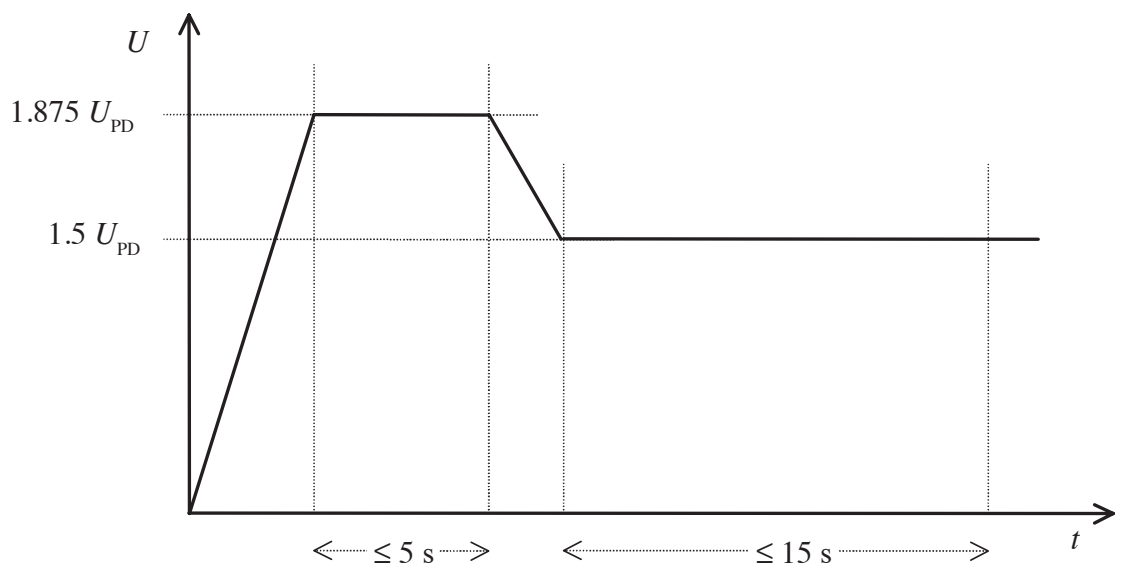

Fig. 9: Voltage profile for the PD tests. The PDs are recorded on the second flat section (15 s) as specified in IEC 61800-5. Image taken from [6]. 


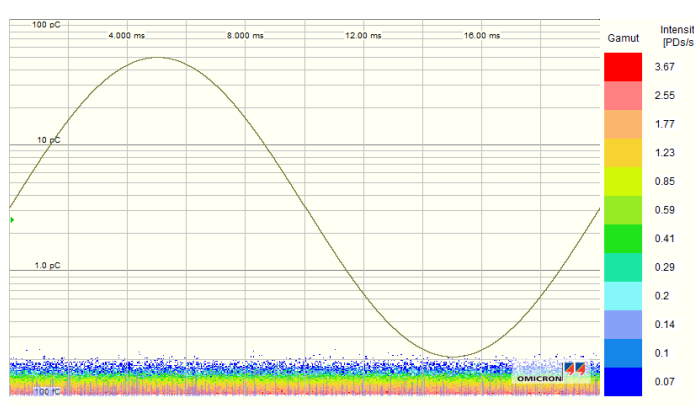

(a)

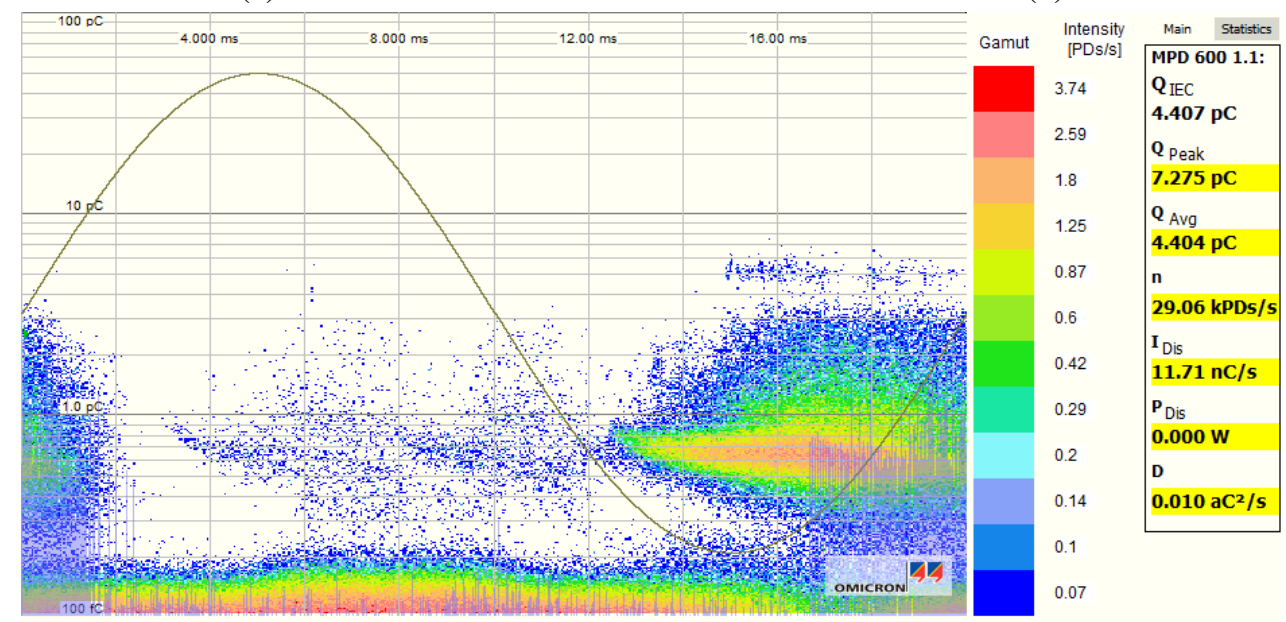

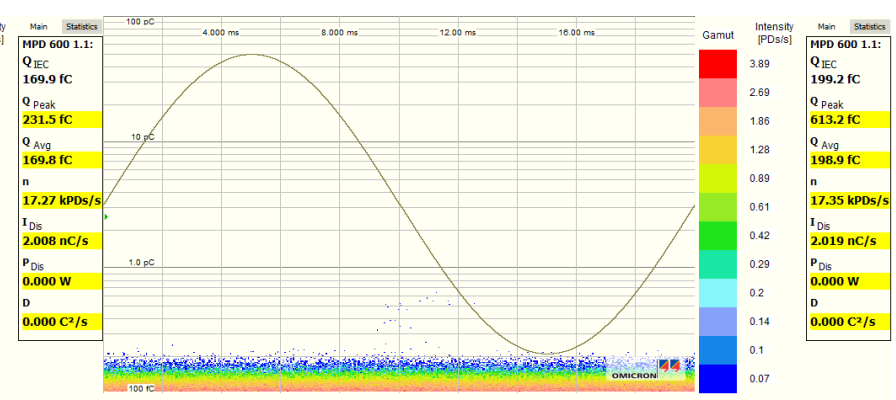

(b)

(c)

Fig. 10: (a) PD histogram for test $1, U_{\mathrm{PD}}=1 \mathrm{kV}_{\mathrm{ac}}$, (b) PD histogram for test $2, U_{\mathrm{PD}}=9.3 \mathrm{kV}_{\mathrm{ac}}$ and (c) PD histogram for test $3, U_{\mathrm{PD}}=9.3 \mathrm{kV}_{\mathrm{ac}}$.

A series of tests were performed on the prototype:

Test 1: Two adjacent SMs of the same drawer This is the most critical test at the drawer level. Other tests were performed, but their results are not shown here. A working voltage of $1 \mathrm{kV}_{\mathrm{ac}}$ is considered. The PD test result is presented in Fig. 10a. As it can be read on the image, $Q_{\text {Peak }}=231.5 \mathrm{fC}$, which is below the $10 \mathrm{pC}$ limit specified by the standard.

Test 2: enclosure to sliding rail (at GND) The drawer is designed such that the creepage and clearance distances between one sliding rail and the closest SM has to withstand the full DC link voltage. As is can be seen on Fig. 5b, the sliding rail is mounted on the drawer's vertical panel. The PD test result is presented in Fig. 10b. As it can be read on the image, $Q_{\text {Peak }}=613.2 \mathrm{fC}$, which is way below the $10 \mathrm{pC}$ limit specified by the standard.

Test 3: phase-leg to cabinet frame (at GND) All SMs are connected together (at the same potential), as at any point in time one SM or a group of SMs can be at the maximum potential, depending on the branch insertion index. The PD test result is presented in Fig. 10c. As it can be read on the image, $Q_{\text {Peak }}=7.275 \mathrm{pC}$, which is below the $10 \mathrm{pC}$ limit specified by the standard.

\section{Conclusion}

This paper presented details about the insulation coordination for a MV MMC laboratory prototype, connected to a $10 \mathrm{kV}_{\mathrm{dc}}$ supply with a system voltage of $6.6 \mathrm{kV}_{\mathrm{ac}}$. The UL840 and IEC61800-5-1 standards have been followed and respective clearance and creepage distances have been determined. The converter was partitioned into four zones according to their working voltages. The design phase has been supported by FEM simulations that shown that the E-field magnitude was always below the air 
breakdown level. To verify the design, ac dielectric withstand and PD tests on the real prototype have shown that the IEC61800-5-1 standard requirements were met.

\section{References}

[1] R. Marquardt, A. Lesnicar, and J. Hildinger. "Modulares Stromrichterkonzept für Netzkupplungsanwendungen bei hohen Spannungen”. In: ETG Conference, Proceedings of. 2002.

[2] D. Cottet et al. "Integration Technologies for a Medium Voltage Modular Multi-Level Converter with Hot Swap Capability”. In: Energy Conversion Congress and Exposition (ECCE), 2015 IEEE. Sept. 2015.

[3] A. Christe and D. Dujic. "On the Integration of Low Frequency Transformer into Modular Multilevel Converter”. In: Energy Conversion Congress and Exposition (ECCE), 2015 IEEE. 2015.

[4] A. Christe and D. Dujic. "Galvanically Isolated Modular Converter". In: Power Electronics, IET (accepted) (2016).

[5] UL 840. Insulation coordination: insulation clearances and creepage distances for electrical equipment. 2000.

[6] IEC 61800-5-1. Adjustable speed electrical power drive systems: Part 5-1: safety requirements - electrical, thermal and energy. 2007.

[7] E. Coulinge, A. Christe, and D. Dujic. "Electro-Thermal Design of a Modular Multilevel Converter Prototype". In: PCIM Europe 2016; International Exhibition and Conference for Power Electronics, Intelligent Motion, Renewable Energy and Energy Management; Proceedings of. May 2016, pp. 1-8.

[8] http://www.hundh-mk.com/e/index.php.

[9] https://www.omicronenergy.com/en/products/all/primary-testing-monitoring/mpd-600/\#Description. 